\title{
3-D Ultrasound Elastography Reconstruction Using Acoustically Transparent Pressure Sensor on Robotic Arm
}

\author{
Vincent Groenhuis, Anton Nikolaev, Saskia H.G. Nies, Marcel K. Welleweerd, Leon de Jong, Hendrik H.G. \\ Hansen, Françoise J. Siepel, Chris L. de Korte, Stefano Stramigioli
}

\begin{abstract}
Biopsy of breast lesions only visible on MRI may benefit from patient-specific finite-element modeling of the breast. This requires acquisition of the heterogeneous elasticity distribution. An acoustically transparent pad has been developed which enables strain elastography imaging using ultrasound without need for additional force or pressure sensors. The elasticity of the pad is known a priori while the change in thickness is detectable on ultrasound by edge detection. Based on the calculated pressure distribution across the pad, the pressure in inner tissue can be approximated. The strain is measured using a cross-correlation block matching algorithm and from these measurements the heterogeneous elasticity distribution can be estimated.

The ultrasound probe with pad is moved over the breast surface by a robot arm. At sixteen sites on the surface of one phantom the probe with pad performed an inward push movement perpendicular to the breast surface. The ultrasound scans were post-processed to reconstruct a heterogeneous elasticity map in the local imaging plane. While it is challenging to accurately estimate the stress and strain in deeper tissue, certain features such as the skin can be effectively distinguished from the underlying soft tissue which demonstrates the potential of the system.
\end{abstract}

Index Terms-Ultrasound, Elastography, Robotic scanning

\section{INTRODUCTION}

I $\mathrm{N}$ breast cancer screening, certain suspicious lesions are only detectable on MRI and not by mammography (x-ray), US (ultrasound) or elastography. A biopsy sample of the lesion is required for accurate pathology assessment. The manual MRI-guided biopsy procedure is inaccurate and inefficient: breast tissue may deform due to body movements, muscle contractions and needle-tissue interactions. These changes are often not detectable due to the low sampling frequency. This has led to the launch of research projects focused on the introduction of surgical robotics to overcome the existing limitations and improve the accuracy of biopsies of MRIvisible lesions [1], [2].

The MURAB (MRI and Ultrasound Robotic Assisted Biopsy) project aims to solve these challenges by coregistering MRI images to 3-D US data and 3-D elastography

V. Groenhuis*, M.K. Welleweerd, F.J. Siepel and S. Stramigioli are with Robotics and Mechatronics, University of Twente, Postbus 217, 7500AE Enschede, The Netherlands, phone +31 53489 9111, fax +31534894571

S.H.G. Nies is with Biomechanical Engineering, University of Twente, Enschede, The Netherlands

A. Nikolaev, L. de Jong, H.H.G. Hansen and C.L. de Korte are with Department of Radiology and Nuclear Medicine, Radboud university medical center, Nijmegen, The Netherlands

S. Stramigioli is with ITMO University, Saint Petersburg, Russia

*Corresponding author, e-mail: v.groenhuis@utwente.nl

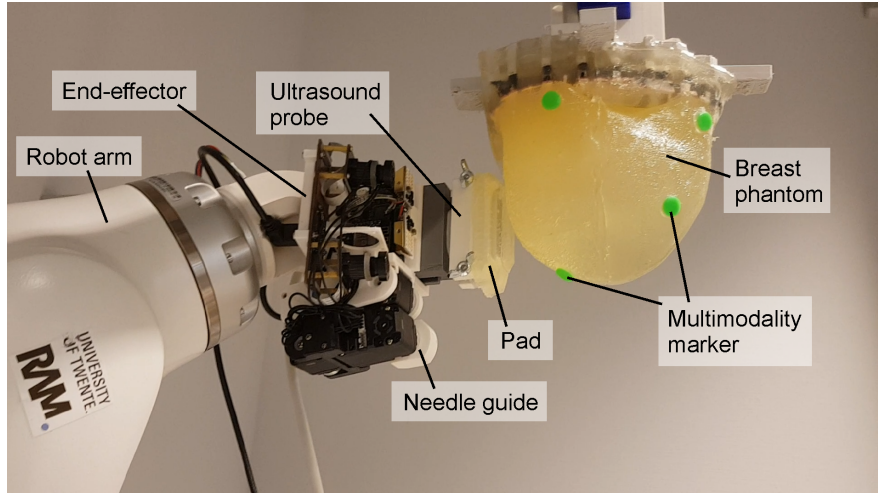

Fig. 1. Experimental setup. The ultrasound probe with pad is mounted on the robot arm's end-effector, the breast phantom hangs in prone position.

maps acquired using a robotic arm. This could enable biopsies of breast lesions only visible on MRI with the accuracy of ultrasound-guided biopsies. A patient-specific finite-element model is constructed to simulate the deformations resulting from probe-tissue and needle-tissue interactions, allowing to optimize the needle insertion trajectory and maximize the chance of a successful biopsy [3], [4]. While a homogeneous elasticity distribution could be assigned to the finite-element model, for more precise simulations a heterogeneous elastography map of the breast would be highly beneficial.

Existing elastography methods generally focus on relative elasticity distributions, highlighting localized variations in stiffness. This is useful when searching for abnormalities in which absolute elasticity values are less relevant, and can be acquired using certain ultrasound systems that support elastography imaging based on e.g. strain imaging [5], [6], [7], [8]. In contrast, our application uses elastography for simulation purposes which requires acquisition of absolute elasticity data which cannot be derived from strain imaging alone. Absolute elasticity maps could be acquired using the acoustic radiation force imaging (ARFI) technique, but this requires specialized equipment with high-power ultrasound transducers [9]. Schneider et al. used an external exciter to generate shear waves in tissue while a transducer acquires vibration phases and volumes, but this works in only one dimension at a time making 2-D and 3-D acquisition cumbersome [10].

In order to acquire heterogeneous absolute elastography data in this research, a special pad has been developed which can be placed over the ultrasound probe. This pad allows to 


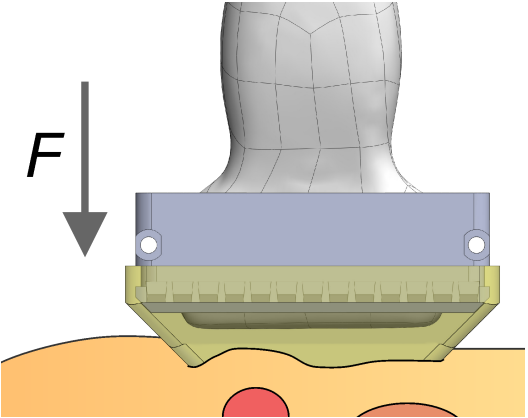

Fig. 2. Working principle of the pressure sensor. The observed pad thickness is related to the local pressure by the known Young's modulus of the pad.

estimate the pressure distribution along the pad based on its thickness, which in combination with strain imaging allows to estimate the absolute elasticity distribution. The robot arm is used to perform consistent and reproducible motions during the automated elastography acquisition procedure, keeping the ultrasound probe in the same plane during acquisition at each site and eventually resulting in a set of reconstructed slices at the correct spatial locations within the breast. The aim of this study is to investigate the feasiblity of the pad and analyze its potential and limitations.

\section{Methods}

In order to acquire elastography data, a phantom is robotically scanned with an ultrasound probe equipped with an acoustically transparent pad. This chapter describes the production of the phantom and pad, the robotic ultrasound scanning procedure based on an optically registered MRI scan, and the post-processing phase involving reconstruction of stress, strain and elasticity. While the effect of gravity has been studied previously [11], [12], it is not taken into account in this research due to the complexities involved.

\section{A. Pad fabrication}

The pad is made of anechoic PVC Plastisol attached to a 3-D printed rigid frame mounted on the US probe. Air bubbles were eliminated as much as possible by degassing the PVC Plastisol in a vacuum chamber before molding. The pad has a nominal thickness of $9 \mathrm{~mm}$ and a flat, rectangular contact surface of $10 \mathrm{~mm} \times 40 \mathrm{~mm}=400 \mathrm{~mm}^{2}$. The edges of the pad are slanted to better stabilize the pad. The stiffness of the pad is a priori estimated by measuring the change in thickness introduced by a known force distributed equally over the pad.

A schematic drawing of the pad is shown in Fig. 2, illustrating the concept that nonhomogeneous elasticity distributions in tissue may result in localized variations in pad thickness which can be measured by ultrasound.

\section{B. Phantom fabrication}

A heterogeneous breast phantom was manufactured using PVC Plastisol, mixed with plasticizer to adjust stiffness and/or mixed with silica gel to adjust echogenicity [11]. The relatively hyperechoic, stiff skin was first produced using a pair of moulds. The inner mould was then removed and hypoechoic soft PVC Plastisol was poured in, adding strands of hyperechoic stiff PVC Plastisol to create semi-random spaghettilike structures to mimick regions of fatty tissue distributed in fibroglandular tissue. Eight green-colored multimodality markers are glued to the breast surface for registration purposes. A rigid frame mimicks the rib cage, allowing to hang the phantom in prone position within the robot's workspace.

\section{MRI scanning, segmentation and registration}

The phantom was previously scanned in a $0.25 \mathrm{~T}$ MRI scanner (G-Scan Brio, Esaote Gmbh, Erlingen, Germany) using a 3D balanced gradient echo sequence. Based on this scan a surface model was constructed and the eight markers segmented separately. After positioning the phantom in prone position it is registered in the world coordinate frame. A KUKA LBR Med (KUKA Gmbh, Augsburg, Germany) robot arm is used of which the end-effector is equipped with a stereo camera (Kayeton CCTV, Shenzhen, China) plus a light source to ensure consistent illumination besides the ultrasound probe with pad [4].

During the registration process the camera system makes one revolution around the breast from a safe distance. The green-colored markers are segmented by thresholding the hue, saturation and brightness components and converted to points in the world coordinate frame by triangulation. As the same marker may be detected multiple times from different angles the resulting pointcloud will contain hundreds to thousands of points. These are grouped based on nearest neighbourhood distances, removing any outliers in the process. When the number of groups equals the number of markers, the markers are co-registered using feature descriptors constructed from the set of intra-marker distances. The optimal rigid transformation is computed so that the phantom is precisely registered in the robot coordinate frame. The mean marker is error calculated and a warning message is displayed when this error exceeds a specific threshold.

\section{Robotic ultrasound scanning}

Based on the segmented and registered MRI scan an automatic ultrasound acquisition trajectory is generated by projecting a series of spirals on the segmented surface of the phantom, taking the workspace and dexterity of the robot arm into account. During this trajectory the ultrasound probe is kept orthogonal to the breast surface, but does not yet make contact. On sixteen sites a gradually increasing force is exerted on the breast while the probe is constrained to move in the ultrasound plane only. The forces are measured and regulated by the robot arm itself, operating in impedance control mode with low spring stiffness in the imaging direction and maximum stiffness in all other directions. The maximum force in the imaging direction is tuned to the stiffness of the specific breast or phantom, in this case $15 \mathrm{~N}$. This results in the compression of tissue in the US imaging plane, locally displacing the skin by up to two centimetres. The ultrasound scans are acquired using the Siemens P500 system with L10-5v probe (Siemens Healthineers AG, Erlangen, Germany). 


\section{E. Strain reconstruction}

The reference image of the sequence is the one in which the probe just makes contact with the surface and the stress and strain are approximately zero. A small bias in stress or strain is acceptable as relative changes are used in elasticity calculation. The subsequent frames are used to calculate incremental tissue displacements using a cross-correlation based block matching algorithm. This algorithm subdivides each scan in blocks of $20 \times 20$ pixels for which matching blocks are found within specific search windows in the reference frame. If such a match is found with high confidence, a displacement vector is associated to this block. Gaps, if any, are filled by replicating vectors from nearest neighbours and averaged if necessary. Differences in displacement vectors among neighbouring blocks are a measure of strain and converted to a strain map.

\section{F. Stress reconstruction}

The skin-pad boundary is estimated by edge detection of the US scans. As the pad is not totally anechoic, several techniques were applied including Gaussian smoothing in horizontal direction and elimination of small blobs formed by air bubbles in the pad. The topmost edge spanning the full image width is then robustly classified as the pad-skin boundary. By measuring the local pad thickness in each scan and comparing it to the reference scan thickness, the local pad strain is estimated. As the Young's modulus of the pad is known, the stress distribution inside the pad is derived. In this calculation it is assumed that the pad is sufficiently elastic and the breast surface is sufficiently smooth, i.e. no discontinuities are present.

The stress distribution across the pad needs to be propagated to the full US scan region. As the total force is being distributed over larger cross-sectional areas, the pressure is attenuated at increasing depth. The exact stress distribution depends on several factors including the breast shape, amount of deformation and material properties such as Young's modulus, Poisson ratio, visco-elastic and non-linear material characteristics. If one assumes an infinitely long and thin pad placed on a perfectly linear-elastic, homogeneous block phantom, isosurfaces of equal stress will be approximately half-cylinder shaped with the stress being inversely proportional to the width of the isosurface area due to the balance of forces acting on these isosurfaces. This gives a crude, but mathematically valid approximation of the propagation of stress through tissue as far as the depth stays well below the pad length. This combined with earlier experiments and simulations on homogeneous block phantoms a linear attenuation coefficient with horizontal smoothing factor is estimated.

\section{G. Elasticity reconstruction}

From the stress and strain maps a quantitative measure of the elasticity is calculated in every frame, represented in the reference frame. The output elasticity map is calculated by taking the median elasticity in each voxel, removing the outliers. This process is repeated for all sixteen sites on the breast and visualized in a 3-D rendering.
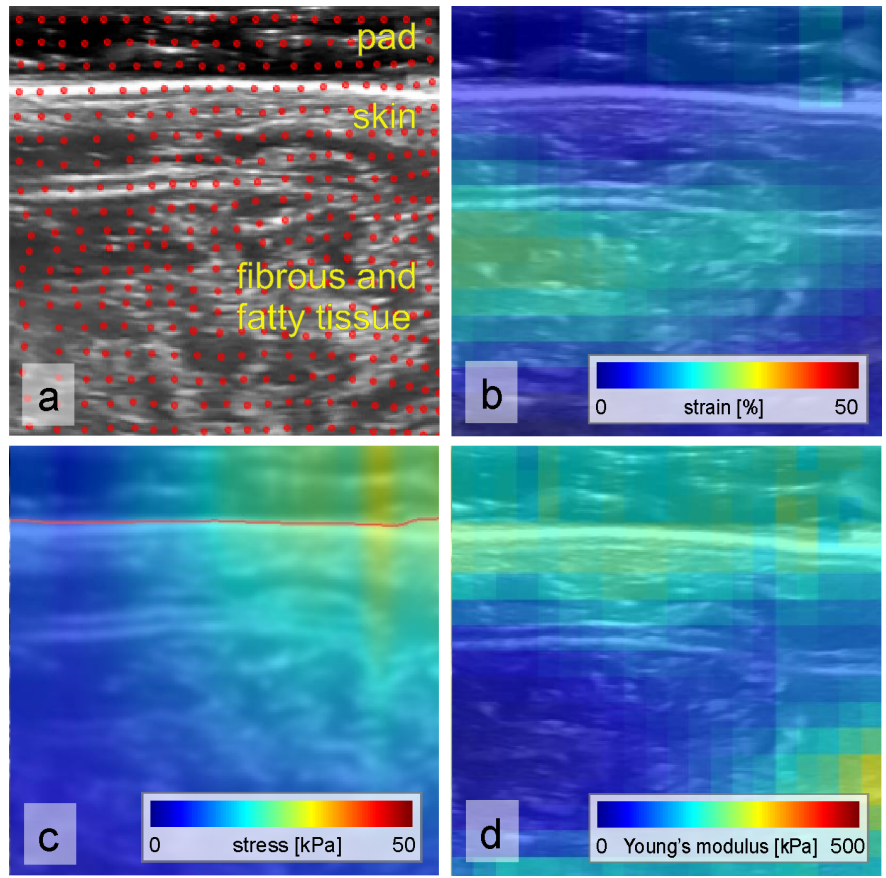

Fig. 3. Post-processing of US data. a) Displacement tracking by block matching algorithm. b) Strain map showing amount of compression. c) Pad boundary and pressure distribution. d) Young's modulus as calculated from stress and strain maps.

\section{RESUlTS AND Discussion}

\section{A. Post-processing US slice for elastography}

The elasticity of the pad was estimated to be approx. $209 \mathrm{kPa}$.

The post-processing graphs of a sample frame of one site are shown in Fig. 3. In Fig. 3a, an ultrasound scan (grayscale) is superimposed with a lattice grid (red) indicating the local displacement of tissue in this frame relative to the reference frame. The (mostly) anechoic pad can be clearly distinguished from the hyperechoic skin (white). In Fig. 3b, the strain is calculated from the compression in vertical direction which relates to the change in vertical distance between the dots in the lattice grid relative to the reference frame. In Fig. 3c, the automatically segmented pad-skin boundary is shown in red. At one point it was measured to be $8.8 \mathrm{~mm}$ in the reference frame and $8.0 \mathrm{~mm}$ in this sample frame, resulting in a strain of $\frac{8.8-8.0 \mathrm{~mm}}{8.0 \mathrm{~mm}}=0.10$ and a stress of $0.10 \cdot 209 \mathrm{kPa}=21 \mathrm{kPa}$. This pressure is propagated downwards using a linear attenuation coefficient and horizontally smoothed with other columns. In Fig. 3d, the stress and strain maps are combined to calculate the elasticity distribution of the sample frame. It can be observed that the skin has a higher Young's modulus (approx. $250 \mathrm{kPa}$ ) than most of the deep tissue (up to $150 \mathrm{kPa}$ ) which is consistent with the fabrication process of the phantom.

\section{B. 3-D visualization of elastography acquisitions}

Fig. 4 shows a 3-D rendering of the breast phantom. The square-shaped colored surfaces represent the elasticity measurement results at the sixteen sites on the phantom. It is observed that the stiffness of skin and deep tissue are 


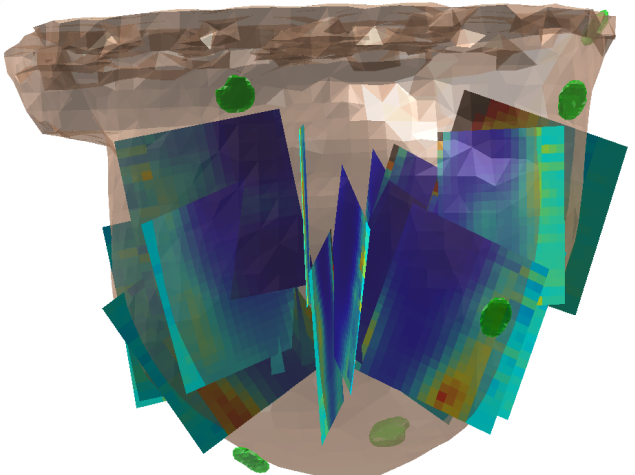

Fig. 4. 3-D rendering of the phantom with sixteen elasticity measurement slices.

consistent across the different slices, and that some noise is present in specific areas of the elasticity maps at certain sites.

\section{Discussion}

The correctness of the reconstructed elasticity maps highly depend on the quality of the acquired stress and strain maps.

The stress distribution along the pad is calculated from the observed change in pad thickness. As shown in Fig. 3a, the pad is not free of scattering particles such as air bubbles due to manufacturization effects, negatively affecting the thickness observations. Also, the pad's surface is straight while the phantom has a rounded surface, implying an uneven stress distribution in the reference frame. The apparent variation in pad stiffness in Fig. 3d is caused by differences in pad strain measurements in Fig. $3 b$ and Fig. 3c.

The stress and strain maps are calculated relative to the reference frame in which both stress and strain are assumed to be zero, but in reality the bias cannot be ignored. Furthermore, the estimation of stress in deep tissue is a significant aspect which needs to be investigated more thoroughly.

The overall stiffness of the phantom (order of $150 \mathrm{kPa}$ ) is significantly higher than that of a natural breast (order of $10-50 \mathrm{kPa}$ ) [13]. This is partly due to the limited effective stiffness range of PVC Plastisol, and also due to the particular measurement setup with the phantom free-hanging in prone position without support at the other side of the breast. The use of a softer phantom or natural breast would result in larger displacements under similar stress, requiring optimization of algorithms to properly estimate stress and strain in the different acquisitions.

The end result is a 3-D rendering of the phantom superimposed with sixteen 2-D elasticity slices as shown in Fig. 4. It can be observed that the skin is consistently stiffer than the deeper tissue, which is consistent with the produced phantom. The sixteen slices are not evenly distributed over the full phantom. The robotic path planning algorithm and acquisition method should be improved to better cover the tip the breast and the innermost parts as well.

\section{CONCLUSION}

This research shows that it is possible to quantitatively acquire elastography data using a ultrasound probe equipped with an acoustically transparent pad. Measurements on a PVC phantom show consistent results across different sites of the phantom. The proposed method is a promising technique for acquiring elastographic data and needs to be researched further to optimize and cross-validate the results. Additional ex-vivo and/or in-vivo experiments should be conducted to gather more reference data for calibration, and to assess the accuracy of the described method in comparison to other techniques.

\section{ACKNOWLEDGMENT}

This project has received funding from the European Union's Horizon 2020 research and innovation programme under grant agreement No 688188 .

\section{REFERENCES}

[1] V. Groenhuis, F.J. Siepel, M.K. Welleweerd, J. Veltman, S. Stramigioli, "Sunram 5: An MR Safe Robotic System for Breast Biopsy." In Proceedings of Hamlyn Symposium on Medical Robotics, London, United Kingdom, pp. 82-83, June 2018.

[2] D. Stoianovici, C. Jun, S. Lim, P. Li, D. Petrisor, S. Fricke, K. Sharma K. Cleary, "Multi-imager compatible, MR safe, remote center of motion needle-guide robot." IEEE Transactions on Biomedical Engineering, 65(1), pp. 165-177, 2017.

[3] F. Visentin, V. Groenhuis, B. Maris, D. Dall'Alba, F.J. Siepel, S. Stramigioli, P. Fiorini, "Iterative simulations to estimate the elastic properties from a series of MRI images followed by MRI-US validation." Medical \& biological engineering \& computing, 57(4), 913-924, 2019.

[4] V. Groenhuis, E. Tagliabue, M.K. Welleweerd, F.J. Siepel, J.D. Munoz Osorio, B.M. Maris, U. Zimmermann, P. Fiorini, S. Stramigioli, ”Deformation Compensation in Robotically-Assisted Breast Biopsy." Abstract from 11th International Conference on Information Processing in ComputerAssisted Interventions (IPCAI), Munich, Germany, 2020.

[5] J.E. Brandenburg, S.F. Eby, P. Song, H. Zhao, J.S. Brault, S. Chen, K.N. An, "Ultrasound Elastography: The New Frontier in Direct Measurement of Muscle Stiffness." Archives of Physical Medicine and Rehabilitation 95(11), 2207-2219 2014.

[6] G.A. Hendriks, C. Chen, H.H. Hansen, C.L. de Korte, "Quasi-static elastography and ultrasound plane-wave imaging: The effect of beamforming strategies on the accuracy of displacement estimations." IEEE International Ultrasonics Symposium (IUS), 2017.

[7] R.G. Barr, A. De Silvestri, V. Scotti, F. Manzoni, C. Rebuffi, C. Capittini, C. Tinelli, "Diagnostic performance and accuracy of the 3 interpreting methods of breast strain elastography: a systematic review and metaanalysis." Journal of Ultrasound in Medicine, 38(6), 1397-1404, 2019.

[8] R.G. Barr, S.I. Nikolov, "Use of a Real-Time Stress Map for Assessment of Applied Stress for Strain Elastography: Utility in Training and Computation of Strain Ratios." Journal of Ultrasound in Medicine, 38(11), 2999-3005, 2019

[9] K. Rifai, J. Cornberg, I. Mederacke, M.J. Bahr, H. Wedemeyer, P. Malinski, H. Bantel, B. Boozari, A. Potthoff, M.P. Manns, M. Gebel, "Clinical feasibility of liver elastography by acoustic radiation force impulse imaging (ARFI)", Digestive and Liver Disease, Vol 43(6), pp. 491-497, 2011

[10] C. Schneider, A. Baghani, R. Rohling, S. Salcudean, "Remote ultrasound palpation for robotic interventions using absolute elastography." In International Conference on Medical Image Computing and ComputerAssisted Intervention, pp. 42-49. Springer, Berlin, Heidelberg, 2012.

[11] V. Groenhuis, F. Visentin, F.J. Siepel, B.M. Maris, D. Dall'alba, P. Fiorini, S. Stramigioli, "Analytical derivation of elasticity in breast phantoms for deformation tracking." International journal of computer assisted radiology and surgery (IJCARS), 13(10), pp. 1641-1650, 2018.

[12] V. Groenhuis, "Robotic systems for breast biopsy using MRI and ultrasound imaging: Optimal guidance to target lesion in deformable tissue." Doctoral dissertation, University of Twente, 2020.

[13] T.P.B. Gamage, P.M. Nielsen, M.P. Nash, "Clinical applications of breast biomechanics." In Biomechanics of living organs, Academic Press, pp. 215-242, 2017 